\title{
A Scale-Dependent Dynamic Model for Scalar Transport in the Atmospheric Boundary Layer
}

\author{
Fernando Port-Agel and Qiao Qin \\ Department of Civil Engineering and St Anthony Falls Laboratory, \\ University of Minnesota, Minneapolis, MN 55414 \\ fporte@tc. umn.edu
}

\begin{abstract}
A key factor in large-eddy simulations (LES) of the atmospheric boundary layer is our limited ability to account for the physics that are not explicitly resolved in the simulations. These subgrid-scale (SGS) processes are particularly important in the near-ground region, where the characteristic eddy size is typically on the order of (or smaller than) the grid size. Numerical simulations and field experiments were performed to address open issues in SGS modeling. A new scale-dependent dynamic model was developed and used to optimize the value of the SGS model coefficient in the eddy-diffusion model at every position and time step based on the resolved field. Simulations with the scale-dependent dynamic model yield the expected trends of the coefficient as function of position, scale, and atmospheric stability. Furthermore, the new model gives improved predictions of mean profiles and turbulence spectra as compared with the traditional eddy-diffusion and dynamic models. Scale dependence of the model coefficient is further explored using high-resolution wind velocity and temperature measurements obtained in the surface layer using arrays of twelve three-dimensional sonic anemometers.
\end{abstract}

V.N. Alexandrov et al. (Eds.): ICCS 2001, LNCS 2074, p. 1062, 2001.

(c) Springer-Verlag Berlin Heidelberg 2001 